\title{
Dapsone Gel
}

National Cancer Institute

\section{Source}

National Cancer Institute. Dapsone Gel. NCI Thesaurus. Code C66931.

The 5\% gel preparation of dapsone, a synthetic derivative of diamino-sulfone with antiinflammatory and anti-bacterial properties. As a structural analog of p-aminobenzoic acid (PABA), dapsone inhibits dihydropteroate synthase (DHPS), an enzyme important in folate synthesis, resulting in a depletion of the folate pool and a reduction in the amount of thymidylate available for DNA synthesis. Although the exact mechanism through which dapsone exerts its anti-inflammatory activity has yet to be fully elucidated, this agent interferes with the activation and oxidative damage of myeloperoxidase in neutrophils and inhibits the integrin-mediated adherence and chemotaxis of neutrophils. Dapsone's anti-inflammatory activities may contribute to the effects seen upon topical administration. 